\title{
Primary and predicted secondary structures of the Actinomadura R39 extracellular DD-peptidase, a penicillin-binding protein (PBP) related to the Escherichia coli PBP4
}

\author{
Benoît GRANIER,* Colette DUEZ,* Sophie LEPAGE,* Serge ENGLEBERT,* Jean DUSART,*‡ \\ Otto DIDEBERG, ${ }^{*}$ Jozef VAN BEEUMEN, $\dagger$ Jean-Marie FRERE* and Jean-Marie GHUYSEN* \\ ${ }^{*}$ Centre d'Ingénierie des Protéines, Université de Liège, Institut de Chimie, B6, B-4000 Sart Tilman (Liège 1), and †Laboratorium \\ voor Microbiologie en Microbiële Genetica, Rijksuniversiteit-Gent, K.L. Ledeganckstraat 35, B-9000 Gent, Belgium
}

\begin{abstract}
As derived from gene cloning and sequencing, the 489-amino-acid DD-peptidase/penicillin-binding protein (PBP) produced by Actinomadura R39 has a primary structure very similar to that of the Escherichia coli PBP4 [Mottl, Terpstra \& Keck (1991) FEMS Microbiol. Lett. 78, 213-220]. Hydrophobic-cluster analysis of the two proteins shows that, providing that a large 174-amino-acid stretch is excluded from the analysis, the bulk of the two polypeptide chains possesses homologues of the active-site motifs and secondary structures found in the class A $\beta$-lactamase of Streptomyces albus $\mathrm{G}$ of known three-dimensional structure. The 174-amino-acid insert occurs at equivalent places in the two PBPs, between helices $\alpha 2$ and $\alpha 3$, away from the active site. Such an insert is unique among the penicilloyl serine transferases. It is proposed that the Actinomadura R39 PBP and E. coli PBP4 form a special class, class C, of low- $M_{\mathrm{r}}$ PBPs/ DD-peptidases. A vector has been constructed and introduced by electrotransformation in the original Actinomadura R39 strain, allowing high-level expression and secretion of the DD-peptidase/PBP $\left(250 \mathrm{mg} \cdot \mathrm{l}^{-1}\right)$. The gene encoding the desired protein is processed differently in Actinomadura R39 and Streptomyces lividans. Incorrect processing in Streptomyces lividans leads to a secreted protein which is inert in terms of DD-peptidase activity and penicillin-binding capacity.
\end{abstract}

\section{INTRODUCTION}

The $\beta$-lactamases, the low- $M_{\mathrm{r}}$ penicillin-binding proteins (PBPs)/DD-peptidases and the penicillin-binding domains of the bifunctional high- $M_{\mathrm{r}}$ PBPs are believed to form a superfamily of evolutionarily related penicilloyl serine transferases [1]. However, only a few $\beta$-lactamases of class $\mathrm{A}$, one $\beta$-lactamase of class $\mathrm{C}$ and the Streptomyces R61 low- $M_{\mathrm{r}}$ PBP/DD-peptidase of class B are of known three-dimensional structures [1]. Hence the case of a divergent evolution is mainly supported by predictional studies.

Hydrophobic-cluster analysis [2,3] is a powerful method for analysing proteins that are weakly related in the primary structure. By using this method, it has been shown that, among the low- $M_{\mathrm{r}}$ PBPs/DD-peptidases, the Streptomyces K15 PBP, the Escherichia coli PBP5 and the Bacillus subtilis PBP5 of class A have similarity in the polypeptide folding with the class $A \beta$ lactamases [4]. Recently, Mottl et al. [5] reported that the $E$. coli PBP4 has a type of primary structure which is unique among the penicillin-interactive proteins. Gene cloning and sequencing has shown that the secretory PBP produced by a very taxonomically distant species, Actinomadura R39, has a primary structure which is extremely similar to that of PBP4. It is proposed that these two proteins form a distinct class, class C, of low- $M_{r}$ PBPs/DD-peptidases.

\section{MATERIALS AND METHODS}

\section{Bacterial strains, plasmids and media}

Actinomadura R39 (from this laboratory) was grown at $28^{\circ} \mathrm{C}$ with vigorous orbital shaking in tryptone soya broth (Oxoid). Plasmids pBR322 and pBR325 were used for cloning experiments in Escherichia coli HB101. Growth was at $37^{\circ} \mathrm{C}$ in Luria-Bertani medium. Streptomyces lividans TK24 and plasmids pIJ486 and pIJ702 were from the John Innes Institute, Norwich, U.K.; $S$. lividans was grown in MYEME medium [6] and recombinant Actinomadura R39 was grown in TAU medium [7]. The R2YE medium [8] was also used.

\section{Recombinant DNA techniques, radioactive oligonucleotide probe} and nucleotide sequence

The Actinomadura chromosomal DNA was prepared as described in [8], and the recombinant DNA techniques were performed essentially as described in [9].

The sequence of the 58-amino-acid $N$-terminal region of the Actinomadura R39 PBP was determined on $3.2 \mathrm{nmol}$ of protein (purified as described in [10]), using a 477-A pulsed liquid sequenator with on-line analysis of the amino acid phenylthiohydantoin derivatives and a 120-A analyser (Applied Biosystems, Forster City, CA, U.S.A.). On the basis of these data and the known Actinomycetes codon usage, and by using the PCR procedure [11], a 109 bp DNA segment was prepared and, from this, the non-degenerated 27-mer probe $3^{\prime}$-CGG-CACAGC-CCC-CAC-CAG-CAG-CAC-CTG-5' was synthesized. This probe was complementary to the nucleotide sequence

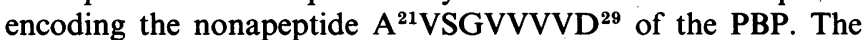
probe was labelled with $\left[\gamma^{32} \mathrm{P}\right] \mathrm{ATP}$ and served to screen gene libraries by hybridization $[12,13]$.

DNA segments cloned into M13 vectors were sequenced by the dideoxynucleotide-chain-termination method [14]. Zones of base compression due to high GC content were resolved using dITP instead of dGTP (Sequenase kit; USB, Cleveland, OH, U.S.A.). Codon usage was analysed with Staden's program [15] and Fickett's test [16] using the Actinomadura R39 $\beta$-lactamase [17] as reference.

\section{Immunological screening}

Rabbit anti-(Actinomadura R39 DD-peptidase/PBP) antiserum was prepared by Gamma S.A. (Tavier, Belgium) and was

\footnotetext{
Abbreviations used: PBP, penicillin-binding protein; ORF, open reading frame.

$\ddagger$ To whom correspondence should be addressed.

The nucleotide sequence data reported will appear in the EMBL, GenBank and DDBJ Nucleotide Sequence Databases.
} 


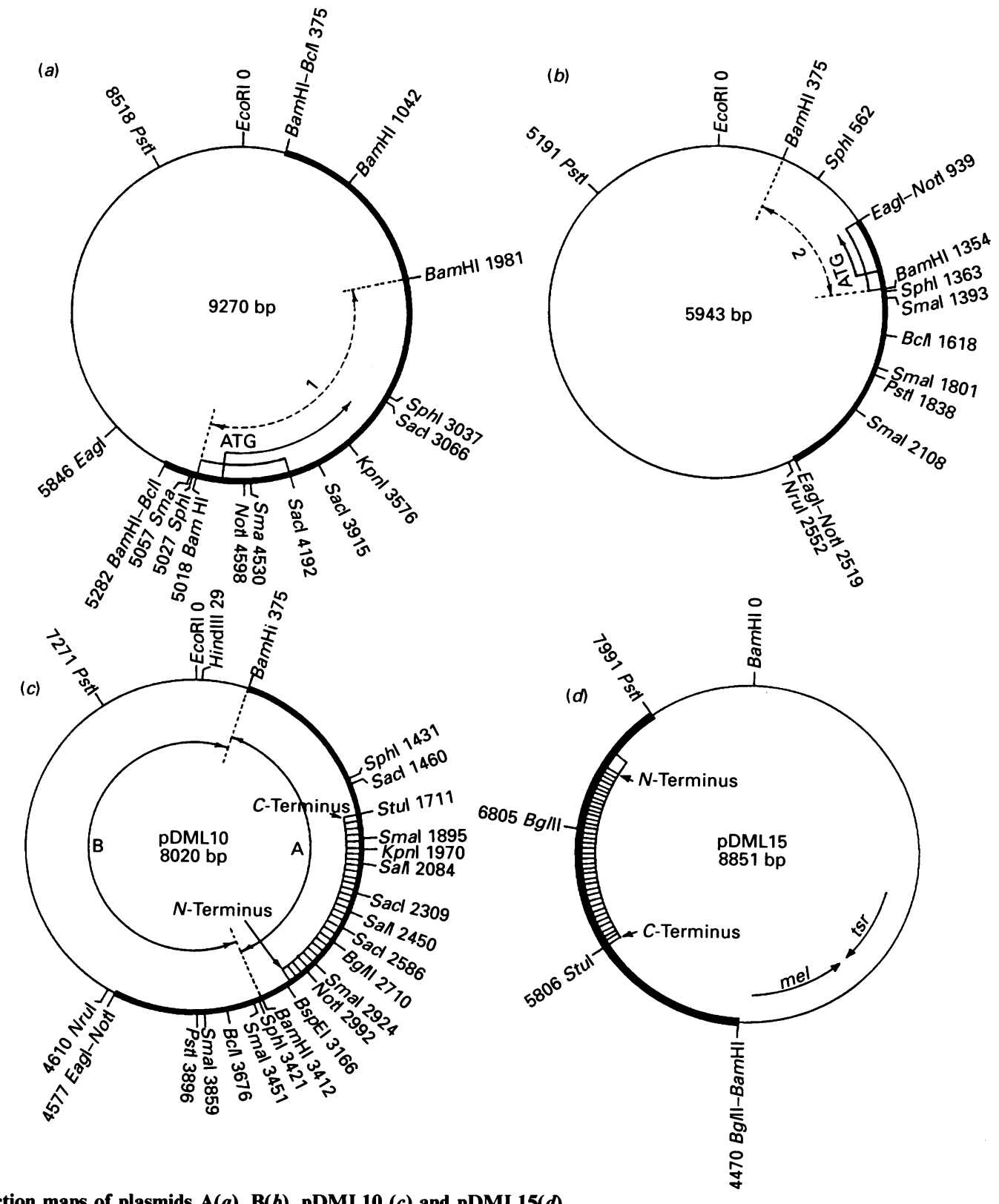

Fig. 1. Restriction maps of plasmids A(a), B(b), pDML10 (c) and pDML15(d)

Inserts are shown in heavy lines. The open boxes in plasmids A and B indicate the hybridizing regions. The position of the initiation codon ATG and the orientation of the ORF in plasmids A and B are indicated. The hatched line in pDML10 and pDML15 specifies the Actinomadura R39 DD-peptidase/PBP-encoding gene. Abbreviations: $m e l$, tyrosinase; $t s r$, thiostrepton resistance.

used to screen PBP-secretory clones. Bio-Rad Immuno Blot Alkaline Phosphatase Assay Systems were employed.

\section{DD-Peptidase activity}

Measurement of the DD-carboxypeptidase activity was performed on Acetyl $_{2}$-L-Lys-D-Ala-D-Ala (release of the $C$-terminal D-Ala) as described in [18].

\section{Electrotransformation}

Cells of Actinomadura R39 [a $48 \mathrm{~h}$ culture made in $25 \mathrm{ml}$ of tryptone soya broth containing $0.5 \%(\mathrm{w} / \mathrm{v})$ glycine] were dispersed by gentle sonication on ice ( 5 bursts of $30 \mathrm{~s} \mathrm{each} ; 20 \mathrm{kHz}$ ), washed by centrifugation $\left(4{ }^{\circ} \mathrm{C} ; 4000 \mathrm{~g} ; 15 \mathrm{~min}\right)$ first with cold water (three times) and then with a $10 \%(\mathrm{v} / \mathrm{v})$ glycerol/water solution (once) and resuspended in $1 \mathrm{ml}$ of glycerol/water. The cell suspension $(100 \mu \mathrm{l})$ and the plasmid (50 $\mathrm{ng}$; see the Results section) were mixed in a $1.5 \mathrm{ml}$ polypropylene tube. The mixture was successively transferred to a $0.2 \mathrm{~cm}$ electroporation cuvette, pulsed once (Cellject, Eurogentec S.A., Liège, Belgium; $40 \mu \mathrm{F}$; $2.5 \mathrm{kV} ; 192 \Omega$ ), supplemented with $1 \mathrm{ml}$ of SOC medium [19] and homogenized with a Pasteur pipette (all these operations were carried out at $4^{\circ} \mathrm{C}$ ). The suspension was shaken at $250 \mathrm{rev} . / \mathrm{min}$ for $1 \mathrm{~h}$ at $37^{\circ} \mathrm{C}$ in a $17 \mathrm{~mm} \times 100 \mathrm{~mm}$ polypropylene tube. The cells were plated on TAU agar medium and, after $10 \mathrm{~h}$ at $28^{\circ} \mathrm{C}$, overlaid with $3 \mathrm{ml}$ of soft agar containing $50 \mu \mathrm{g}$ of thiostrepton $/ \mathrm{ml}$.

\section{Hydrophobic-cluster analysis}

This procedure [2,3] rests upon a representation of the amino acid sequences on an $\alpha$-helical two-dimensional pattern in which 


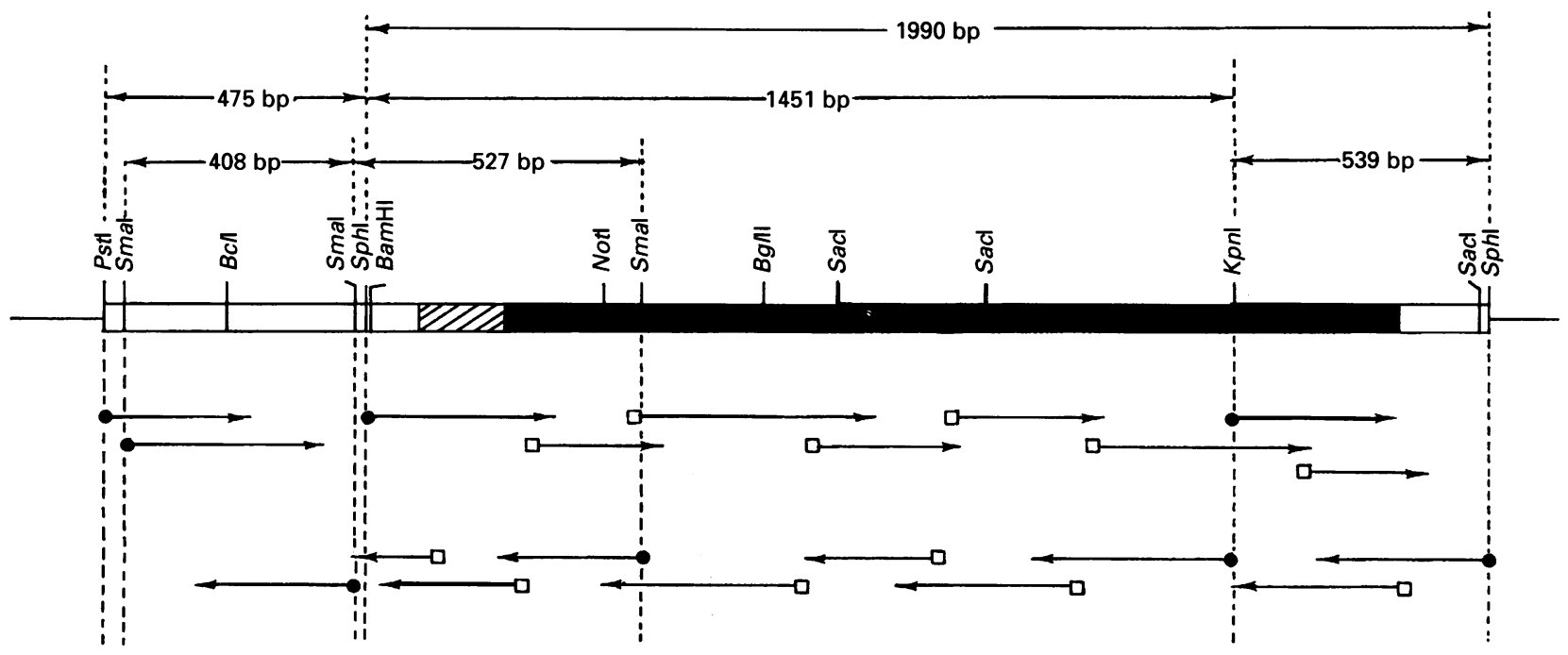

Fig. 2. Strategy of nucleotide sequencing

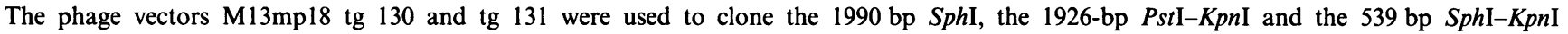
subfragments. The phage vector M13mp10 (cut with SamI and dephosphorylated) was used to clone the 408 bp and 527 bp SmaI subfragments. Nucleotide sequences initiated with the M13 universal primer are marked by ' $O$ '. Those initiated with the DNA probe are marked ' $\square$ '. The arrows indicate the orientation and length of the sequenced segments.

the hydrophobic residues tend to form clusters that usually correspond to the secondary structure elements. Clusters of similar shapes, sizes and relative positions express similarity in the polypeptide folding of the proteins. When compared with methods based only on single-amino-acid property/identity (Goad \& Kanehisa [20]; BESTFIT [21]), the hydrophobic-cluster analysis allows distant information to become more visible and allows deletions or insertions to be introduced more easily between the secondary structures.

\section{RESULTS}

\section{Gene cloning}

The genomic DNA of Actinomadura R39 was cleaved with BamH1, BclI, BglII, NotI, SalI, SphI and NcoI, and the DNA fragments were cloned in pBR322 or pBR325 (for the NcoI library). Among the 4500 ampicillin-resistant E. coli transformants obtained, one clone A from the $B c l$ library and one clone B from the Not I library gave a strong signal with the radioactive probe after washing the filters at $70^{\circ} \mathrm{C}\left(T_{\mathrm{m}}-6^{\circ} \mathrm{C}\right)$. The restriction maps are shown in Figs. $1(a)$ and $1(b)$. The DNA segments responsible for the hybridization signal occurred at the extremity of each of the inserts (open boxes in the Figures). The 500 bp SmaI subfragment from plasmid A and the 800 bp SphI subfragment from plasmid B were prepared, cloned into M13 and sequenced by using the M13 universal primer. Both inserts encoded the $N$-terminal region of the Actinomadura R39 PBP.

In order to allow the orientation of the gene, the $1.45 \mathrm{~kb}$ SphI-KpnI subfragment from plasmid A and the $1 \mathrm{~kb} S p h \mathrm{I}-$ BamHI subfragment from plasmid B were prepared and cloned into M13. Nucleotide sequencing, initiated with the M13 universal primer from the $S p h I$ site, revealed that plasmid A contained the complete PBP-encoding gene (with 4500 bp downstream of the initiation codon ATG), but probably not the complete promoter (with only 370 bp upstream of ATG). Plasmid B contained only part of the PBP-encoding gene (with $300 \mathrm{bp}$ downstream of ATG), but a large $1.25 \mathrm{~kb}$ segment upstream of ATG. Consequently, the BamHI 375-BamHI 1354 DNA segment was excised from plasmid B and replaced by the BamHI
1981-BamH1 5018 DNA segment of plasmid A, yielding pDML10 (Fig. 1c), where the structural gene is very probably preceded by its own promoter.

\section{Gene sequencing and primary structure}

Establishment of the nucleotide sequence of the Actinomadura R39 PBP-encoding gene (using the strategy shown in Fig. 2) revealed a 1614-nucleotide open reading frame (ORF) (Fig. 3). The ORF started with an ATG codon, presented the biased pattern of codon usage typical of Actinomycetes genes and terminated with an Amber codon TGA. This ORF translated into a 538-amino-acid protein precursor whose 49-amino-acid $\mathrm{N}$ terminal region had the features of a long signal peptide. It contained one lysine residue at position -48 , six arginine residues at positions $-40,-38,-37,-33,-29$ and -28 , and a long hydrophobic stretch from Ala-27 to Ala-6. The amino acid sequence of the protein from Arg-1 to Ser-38 was that of the $N$ terminal region of the mature Actinomadura R39 DD-peptidase/ PBP.

\section{Expression of the cloned gene in S. lividans and Actinomadura R39}

The BamHI 375-PstI 3896 DNA segment, containing the complete structural gene and the $590 \mathrm{bp}$ upstream region was excised from pDML10 and ligated to the Streptomyces highcopy-number plasmid pIJ702 (previously cleaved with BglII and PstI and treated with bacterial alkaline phosphatase). The resulting plasmid pDML15 (Fig. 1d) was used to transform $S$. lividans TK24 protoplasts. Among the transformants (selected on R2YE agar plates containing $6 \mu \mathrm{g}$ of thiostrepton $/ \mathrm{ml}), S$. lividans BG2 was the best producer of the expected protein, as evidenced by the immunological test. However, when $S$. lividans BG2 was cultivated in MYEME liquid medium, the secreted protein, though reacting with the anti-(Actinomadura R39 DDpeptidase/PBP) antiserum, lacked both DD-peptidase activity and penicillin-binding capacity. Moreover, it migrated on SDS/ PAGE with an apparent molecular mass about $3 \mathrm{kDa}$ larger than that of the original DD-peptidase/PBP.

In spite of these abnormalities, pDML15 was re-isolated from $S$. lividans BG2 and introduced in the Actinomadura R39 strain 


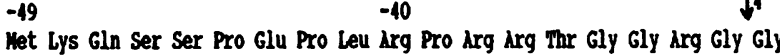

$-40$

$\downarrow 4$

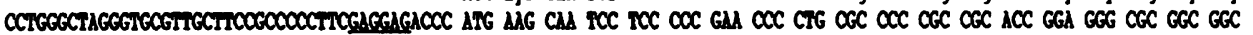

$-30 l^{3} \quad \downarrow^{2} \quad-20 \quad-10 \quad \downarrow 1$

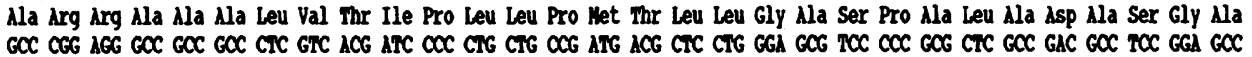

11002000 COC CTC AOC GAA CIG COC GAG GAC ATC GAC GCC ATC CTC GAC GAC COC GCA CTC GAC GCC GCC GNC TCC GCG GTC GTC GTC GTC GAC ACC

$-50$

60 Ala Thr Gly Glu Glu Leu Tyr Ser Arg Asp Gly Gly Glu Gln Leu Leu Pro Mla Ser Asn Met lys Leu Phe Thr Ala Ala Ala Ala Leu

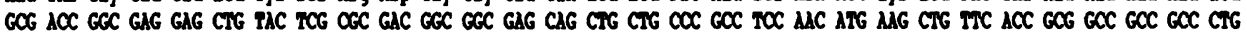

70

90 Glu Val Leu Gly Ala Asp His Ser Phe Gly Thr Glu Val Ala Ala Glu Ser Ala Pro Gly Arg Arg Gly Glu Val Gin Asp Leu Tyr Leu

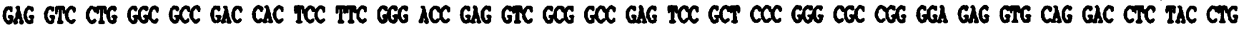

100 110

Val Gly arg Gly Asp Pro Thr Leu Ser Ala Glu Asp Leu Asp Mla Het Mla Ala Glu Val Mla Mla Ser Gly Val Arg thr val Arg Gly GIG GGC COG GCC GAC COE ACE CTC TCC GOC GAG GAC CTC GAC GOC ATC GOC GOC GAG GTC GCC GCC TCC GCG GTC OCC ACC GTC AGG GGC

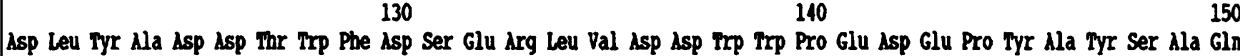

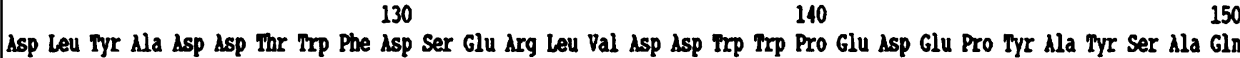
GAC CTE IAC GCC GAC GAC ACG TGG TTC GAC TCC GAG COG CTC GIG GAC GAC TEG TOG COC GAC GAC GAG CCC TAC GCC TAC TCE GCC CAC

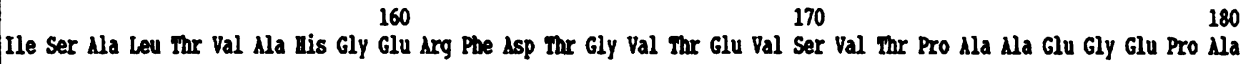

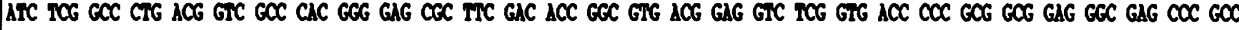

190

200

210

Asp Val Asp Leu Gly Ala Ala Glu Gly tyr Ala Glu Leu Asp Asn Arg Mla Val thr Gly Mla Ala Gly Ser Ala Asn thr Leu Val Ile GAC GTS GAC CTC GCC GOC GCE GAG GCC IAC GCC GAG CTC GAC AAC COC GCC GTC ACC GCC GOC GCC GCC AGC GOC AAC ACC CTC GTC ATC

220

230

240

Asp Arg Pro Val Gly Thr Asn Thr Ile Ala Val Thr Gly Ser Leu Pro Ala Asp Ala Ala Pro Val Thr Ala Leu Arg Thr Val Asp Glu

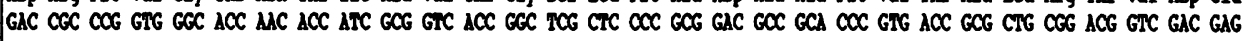

250

260

270

Pro Ala Ala Leu Ala Gly His Leu Phe Glu Glu Ala Leu Glu Ser Asn Gly Val thr Val Lys Gly Asp Val Gly Leu Gly Gly Val Pro

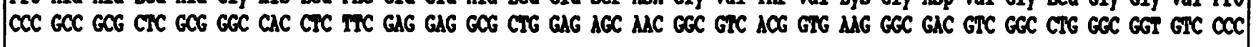

280

300

Nla Asp Trp Gin Asp Ala Glu Val Leu Ala Asp \#is Thr Ser Mla Glu Leu Ser Glu Ile Leu Val Pro Phe Met Lys Phe Ser Asn Asn GCC GAC TGG CAG GAC GOC GAG GTC CTC GCC GAC CAC ACG TOC GOC GAG CTC TCC GAG AIC TIC GTG CCC TTC ATG AAG TIC ACC AAC AAC

310

320

330

Gly His Ala Glu Het Leu Val Lys Ser Ile Gly Gln Glu Thr Nla Gly Ala Gly Thr Trp dsp Ala Gly Leu Val Gly Val Glu Glu Ala

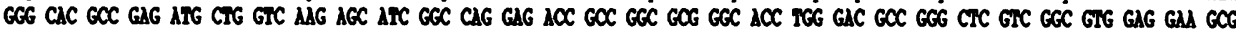

340

350

360

Leu Ser Gly Leu Gly Val Asp Thr Ala Gly Leu Val Leu Asn Asp Gly Ser Gly Leu Ser Arg Gly Asn Leu Val Thr Ala Asp Thr Val

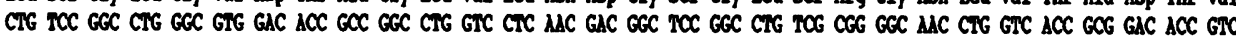

370380 Val Asp Leu Leu Gly Gin Ala Gly Ser Ala Pro Trp Ala Gln Thr Trp Ser Ala Ser Leu Pro Val Ala Gly Glu Ser גsp Pro Phe Val

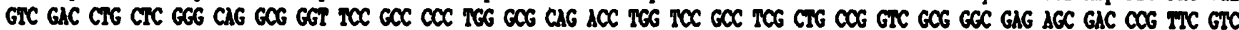

100

110

120

Gly Gly thr Leu Ala Asn Arg Met Arg Gly Thr Ala Ala Glu Gly Val Val Glu Ala Lys Thr Gly Thr het Ser Gly Val Ser Ala Leu

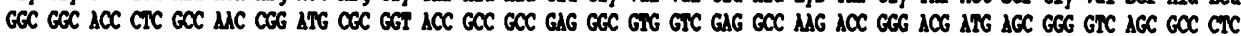

130

140

450

Ser Gly Tyr val Pro Gly Pro Glu Gly Glu Leu dla Phe Ser Ile Val asn isn Gly His Ser Gly Pro Mla Pro Leu Ala Val Gln Asp

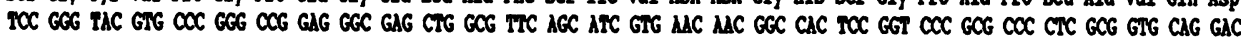

160

Ala Ile Ala Val Arg Leu Mla Glu Tyr Ala Gly nis Gln Ala Pro Glu Gly Ala Arg het Met Arg Gly Pro Val Gln Gly Ser Gly Glu

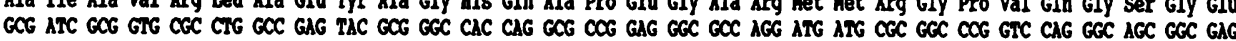

489

Leu Glu Cys Ser Trp Val Gln Ala Cys *k*

CTG GAG TGC TCC TEG GTG CAG GCC TGC TGA CCEGGAGGAGTACCTECCGTCCCECCOTECCOCGAGGG

Fig. 3. Nucleotide sequence of the gene encoding the Actinomadura R39 PBP precursor and deduced amino acid sequence

The site of cleavage by the leader peptidase is indicated by the vertical arrow 1 . Other potential cleavage sites 2,3 and 4 are also indicated (see the text). Ser*, active-site serine. The ${ }^{9^{97}}-\mathbf{P}^{270}$ insert (see the text) is boxed. The putative ribosome-binding site GAGGAG is underlined. 


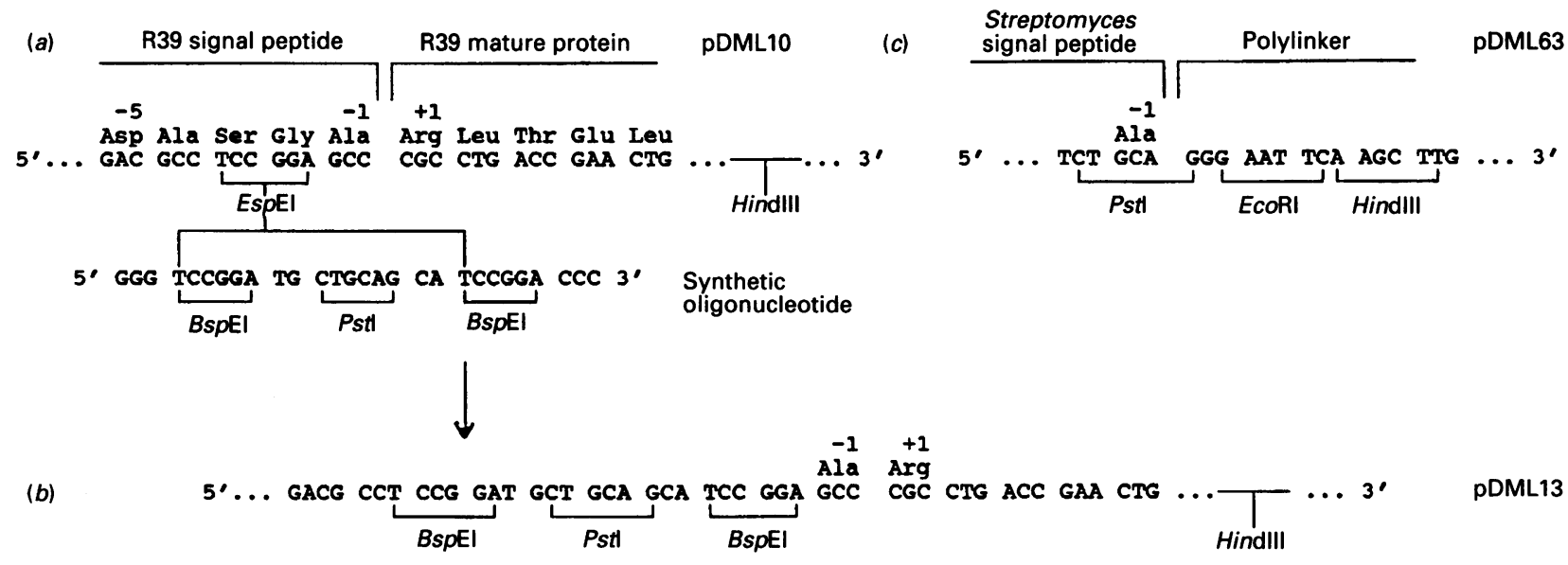

(d)

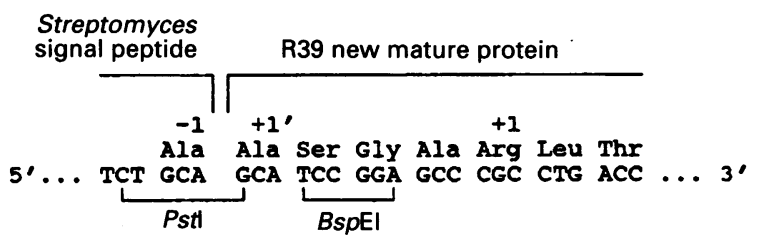

pDML17

Fig. 4. Introduction of the DNA sequence encoding the mature Actinomadura R39 DD-peptidase/PBP in the Streptomyces secretion vector pDML 63: construction of pDML17

(a) A perfect palindromic oligonucleotide was synthesized and allowed to self-hybridize, giving a DNA segment with a PstI site flanked on each side with a BspEI site. The hybrid was digested with $B s p E I$ and inserted in the unique BspEI 3166 site of pDML10 (see Fig. 1c), yielding pDML13 (b), in which the natural signal peptidase cleavage site $\left(\mathrm{Ala}^{-1}-\mathrm{Ala}^{+1}\right.$ ) of the Actinomadura R39 DD-peptidase/PBP is thus preceded by a 17nucleotide sequence containing a PstI and a BspEI site separated by a dinucleotide CA. Digestion of pDML13 with PstI and HindIII liberated a 3100-bp segment that contained the region encoding the mature part of the PBP-encoding gene downstream of a 12-nucleotide sequence coding for Ala, Ser, Gly and Ala. This 3100-bp segment was introduced in the polylinker of pDML63 (c), a Streptomyces high-copy-number secretion vector (see the text), giving rise to pDML17 (d) in which the Streptomyces signal sequence is in phase with the gene encoding the Actinomadura R39 mature protein (with a four-amino-acid $N$-terminal extension).

by electrotransformation, yielding Actinomadura BG3 (note that transformation of the Actinomadura strain by the usual PEGassisted procedure failed and that electrotransformation gave only one transformant in several assays). Under optimal growth conditions in TAU medium, Actinomadura BG3 produced $250 \mathrm{mg}$ of active DD-peptidase/PBP/litre of culture, instead of $15 \mathrm{mg} /$ litre for the original strain (grown under identical conditions). The secreted protein had the 'correct' molecular mass.

A likely hypothesis derived from the above studies was that, in $S$. lividans, incorrect processing of the DD-peptidase/PBP precursor occurred, producing an inactive protein with an extended $N$-terminal region. Examination of the ORF shows that the signal peptide possesses, upstream of the site cleaved in Actinomadura R39 (marked 1 in Fig. 3), other potential cleavage sites (marked 2, 3 and 4) [22]. In particular, site 2 is identical with the $S$. lividans $\beta$-galactosidase signal-peptide-cleavage site [23]. Cleavage of the DD-peptidase precursor at this site would generate a protein with a molecular mass of 52339 (instead of 50053 for the protein processed at site 1).

In order to test the hypothesis, the mature-protein-encoding DNA was introduced into the Streptomyces high-copy-number secretion vector pDML63 (a derivative of pIJ702; A. Brans, M. V. Lenzini, C. Fraipont-Piron \& J. Dusart, unpublished work). This vector possesses Streptomyces transcription, translation and secretion signals, followed by a polylinker (PstI, EcoRI, HindIII, SmaI, XbaI) whose PstI site is in phase with the translation signal. Therefore a PstI site was introduced in the unique BspEI site of the cloned gene, close to the junction between cleavage site 1 and the mature-protein-encoding DNA
(Fig. 1c), the strategy shown in Fig. 4 being followed. The final construction was called pDML17. S. lividans transformed with pDML17 and grown in MYEME medium secreted at least $18 \mathrm{mg}$ of the active DD-peptidase/PBP per litre.

Hydrophobic-cluster analysis: similarity to the $E$. coli PBP4 and structural relatedness with Streptomyces albus $\mathbf{G}$ $\beta$-lactamase of class A

Both the Goad \& Kanehisa algorithm [20] and BESTFIT program revealed high similarity, in the primary structure, between the Actinomadura R39 PBP and the E. coli PBP4 (results not shown). By using the same procedure, no, or only marginal, similarity was observed with the other groups and classes of penicilloyl serine transferases. Hydrophobic-cluster analysis (Fig. 5) confirmed that the Actinomadura R39 PBP and the $E$. coli PBP4 were indeed remarkably similar. Providing that a large deletion was made in the PBPs, the analysis also revealed similarity, in the polypeptide folding, between the two PBPs and the class A Streptomyces albus $\mathrm{G} \beta$-lactamase of known threedimensional structure [24,25]. Fig. 6 shows the amino acid alignments as derived from this analysis.

\section{DISCUSSION}

As a first attempt to obtain the desired Actinomadura R39 gene, a $S$. lividans TK24-pIJ702 cloning system and an immunological screening test were used (B. Granier, unpublished work). Clones were isolated which produced a protein that effectively reacted with the anti-(Actinomadura R39 DD-peptidase/PBP) 


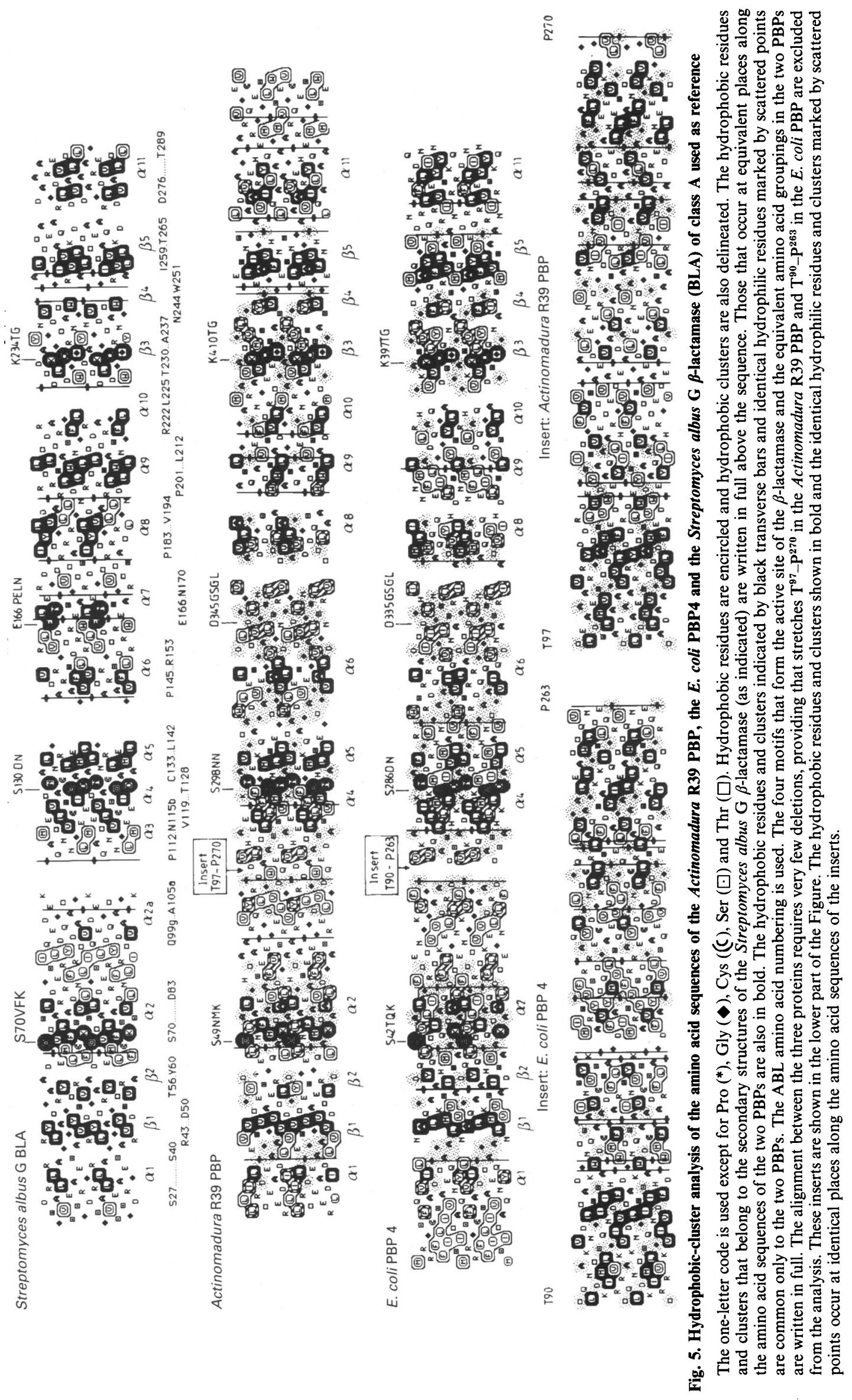




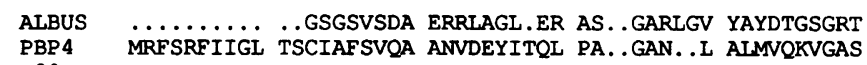
............ RLTELR EDIDAILED. PALEGAVSGV VVVDTATGEE
6 VAYRA...DE LFPMCSVFKT LSSAAVLRDL DRNGEFLSRR ILYTQDDVEQ 104 APAIDYHSQ $Q$ MALPASTQKV ITALAALIQL GPDFRFTTTL ETKGNVENGV 76 LYSRDGG. .E QLLPASNMII FTAAAALEVL GADHSFGTEV AAESAPGRRG 83

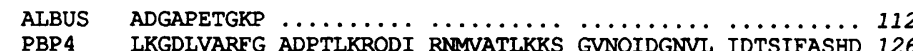
$\begin{array}{lll}\text { PBP4 } & \text { LKGDLVARFG ADPTLKRQDI RNMVATLKKS GVNQIDGNVI IDTSIFASHD } 126 \\ \text { R39 } & \text { EVQDLYLVGR GDPTLSAEDL DAMAAEVAAS GVRTVRGDL ADDTWFDSER } 133\end{array}$
KAPGWPWNDM TOCFSAPPAA AIVDRNCFSV SLYSAPKPG. ..DMAFIRVA 173 LVDDWWPEDE PYAYSAOISA LTVAHGERFD TGVTEVSVTP AAEGEPADVD 183

\section{ALBUS \\ PBP4}

R39

$\begin{array}{llll}\ldots \ldots \ldots \ldots & \ldots \ldots \ldots \ldots & \ldots \ldots \ldots & \ldots \ldots \ldots \\ \text { SYYPVTMFSQ VRTLPRGSAE AQYCELDVV GDLNRFITG CLPQRSEPLP } & 223 \\ \text { LGAAEGYAEL DNRAVTGAAG SANTLVIDRP VGTNTIAVTG SLPADAAPVT } 233\end{array}$

$(130, \mathrm{ABL})$
IAAFAVQDGAS YAGAILKDEL KQAGITWSGT LIRQTOVNEP G.......... ALRTVDEPAA LAGHLFEEAL ESNGVTVKGD VG... LGGVP ADWODAEVLA 280
ALBUS

PBP4

R39

LANGMTVEEL CEVSITASDN CAANLMLREL G..........GPAAVTR 150 $\begin{array}{ll}\text { SKQSAPLHDL LKIMLKKSDN MIADTVFRMI GHARFNVPGT WRAGSDAVRQ } & 318 \\ \text { DHTSAELSEI LVPFMKFSN GHAEMLVKI GQETA.GAGT WDAGLVGVE } 329\end{array}$

$(234, \mathrm{ABL})$

ALBUS LNPRDRRLLT SWLLANTTSG DRFRAGL... . ...PDDWTL GDKTGAGRYG 242

D. R39 . DNELNFISM LPLAGY.DGS LQYRAGLHQA .....GVDGKV SAKTGSLQGV 404 YNL...AGFI TTASGQRMAF VQYLSGYAVE PADQRNRRIP LVRFESRLYK 451 ... PWAQTWS ASLPVAGESD PFVGGTIANR MRGTAAEGVV EAKTGTMSGV 417 SAL...SGYV. .PGPEGELA FSIVNNGHSG P...APLAVQD AIAVRLAEYA 460

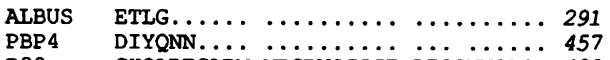

R39 GHQAPEGARM MRGPVQGSE LECSWVQAC. 489

Fig. 6. Amino acid sequence alignment of the Actinomadura R39 PBP, the E. coli PBP4 and the Streptomyces albus G $\beta$-lactamase of class A

The proposed alignment derives from that of Fig. 5. ALBUS, Streptomyces albus G BLA; PBP4, E. coli PBP4; R39, Actinomadura R39 PBP. The motifs that form the $\beta$-lactamase active site are indicated (ABL numbering).

antiserum. This protein had an apparent molecular mass larger than that of the original DD-peptidase/PBP and it was inert in terms of DD-peptidase and penicillin-binding activity, suggesting that the use of a polyclonal antibody might have been misleading. Subsequently, and as described above, the gene was cloned using $E$. coli as host and a nucleotide probe as screening tool, but introduction of the gene in S. lividans, via pDML15, again resulted in the synthesis (in MYEME medium) of a protein which was devoid of enzymic activity and was also about $3 \mathrm{kDa}$ larger than the original DD-peptidase/PBP. However, and at variance with this observation, $S$. lividans TK24 transformed with a secretion vector in which the signal sequence of the Actinomadura gene was replaced by a Streptomyces signal sequence, produced and secreted an active DD-peptidase/PBP of normal size. These studies thus support the view that the protein precursor is processed differently in Actinomadura and in Streptomyces and that the $N$-terminal extension resulting from the incorrect processing in $S$. lividans is sufficient to prevent correct folding of the protein.

Hydrophobic-cluster analysis of the Actinomadura R39 PBP and the E. coli PBP4 and pairwise comparison of the PBPs with the class A Streptomyces albus G $\beta$-lactamase (of known threedimensional structure) leads to the following conclusions. (1) The two PBPs have, along the amino acid sequences, the same typical pattern of distribution of hydrophobic clusters and of hydrophilic residues between the conserved hydrophobic clusters. (2) The peptide stretches $\mathrm{T}^{97}-\mathrm{P}^{270}$ in the Actinomadura $\mathrm{PBP}$ and $\mathrm{T}^{90}-\mathrm{P}^{263}$ in the $E$. coli $\mathrm{PBP}$ have no equivalents in the $\beta$-lactamase. (3) When these adducts are eliminated from the amino acid sequences, the bulk of the polypeptide chain of the two PBPs exhibits similarity with that of the $\beta$-lactamase. (4) Secondary structures equivalent to the $\beta$-lactamase strands $\beta 1$, $\beta 2, \beta 3, \beta 4$ and $\beta 5$ and helices $\alpha 1, \alpha 2, \alpha 4, \alpha 5, \alpha 6, \alpha 8, \alpha 9, \alpha 10$ and $\alpha 11$ are easily identified in the PBPs. (5) Identification in the PBPs of the $S^{*} X X K$ motif (where $S^{*}$ is the essential serine) on $\alpha 2$, the SXN motif between $\alpha 4$ and $\alpha 5$ and the KTG motif on $\beta 3$ is not a matter of controversy. (6) $\mathrm{D}^{345}$ in the Actinomadura PBP and $\mathrm{D}^{335}$ in the $E$. coli $\mathrm{PBP}$ occur at a position comparable with that of $E^{166}$ of the EPELN motif, between $\alpha 6$ and $\alpha 8$, in the $\beta$ lactamase. $E^{166}$ functions as proton abstractor in the attack of the carbonyl carbon of the scissile amide bond of penicillin by the $\gamma$-OH of the $\beta$-lactamase active-site serine residue [25]. The homologues $\mathrm{D}^{345}$ and $\mathrm{D}^{335}$ might play a similar role in the PBPs. Note, however, that another aspartic acid, $D^{337}$ or $D^{327}$, occurs immediately upstream from $D^{345}$ and $D^{335}$. Site-directed-mutagenesis experiments will give answers to the question regarding the essentiality of these acidic side chains in the PBPs. (7) The inserts $\mathrm{T}^{97}-\mathrm{P}^{270}$ in the Actinomadura R39 PBP and $\mathrm{P}^{90}-\mathrm{P}^{263}$ in the $E$. coli PBP4 (whose position is slightly different from that proposed by Mottl et al. [5]) occur between $\alpha 2$ and $\alpha 4$, i.e. on the surface of the proteins. The structure and possible function of the inserts are unknown.

The Actinomadura R39 PBP is a secretory protein. The majority of the $E$. coli PBP4 is exported in the periplasm, at least in the overproducing strains [26]. By analogy to the $\beta$-lactamases, the polypeptide chain of these two water-soluble PBPs terminates immediately or almost immediately after $\alpha 11$. In contrast, the membrane-bound E. coli PBP5 and B. subtilis PBP5 are anchored in the membrane by a short peptide stretch located at the end of a long ( $>100$ amino acids) additional $C$-terminal extension [27].

The Actinomadura R39 PBP and the $E$. coli PBP4 are functionally homologous with respect to the reactions that they catalyse on D-alanyl-D-amino acid-terminated peptides [26-28]. The Actinomadura PBP, however, performs transpeptidation reactions in vitro when incubated in the presence of structured amino acids and peptides [28] and it is extremely susceptible to inactivation by benzylpenicillin (second-order rate constant of acylation of the essential serine: $300,000 \mathrm{M}^{-1} \cdot \mathrm{s}^{-1}$ at $37^{\circ} \mathrm{C}$, as compared with about $7000 \mathrm{M}^{-1} \cdot \mathrm{s}^{-1}$ for the E. coli PBP4) [29].

A last comment must be made. The immediate environment of the active-site serine in the Actinomadura R39 PBP is LPASNMK, not LPASNGV, as proposed previously [30]. It is likely that the 
last two residues were impurities and the isolated peptide was in fact the pentapeptide LPASN.

We thank Mr. D. Klein for technical assistance in DD-peptidase purification. This work was supported, in part, by the Belgian programme on Interuniversity Poles of Attraction initiated by the Belgian State, Prime Minister's Office, Science Policy Programming (PAI $\mathrm{n}^{0} 19$ ), Actions concertées with the Belgian Government (conventions 86/91-90 and 89/94-130), the Fonds de la Recherche Scientifique Médicale (contract $\mathrm{n}^{0} 3.4537 .88$ ), a Convention tripartite between the Région wallonne, SmithKline Beecham, U.K., and the University of Liège, and the Belgian Incentive Program on Fundamental Research in Life Sciences (contract BIO 22). C.D. and J.D. are Chercheurs qualifiés of the Fonds National de la Recherche Scientifique, Brussels.

\section{REFERENCES}

1. Ghuysen, J. M. (1991) Annu. Rev. Microbiol. 45, 37-67

2. Gaboriaud, C., Bissery, V., Benchetrit, T. \& Mornon, J. P. (1987) FEBS Lett. 224, 149-155

3. Henrissat, B., Saloheimo, M., Lavaitte, S. \& Knowles, J. K. C. (1990) Proteins Struct. Funct. Genet. 8, 251-257

4. Palomeque-Messia, P., Englebert, S., Leyh-Bouille, M., NguyenDistèche, M., Duez, C., Houba, S., Dideberg, O., Van Beeuman, J. \& Ghuysen, J. M. (1991) Biochem. J. 279, 223-230

5. Mottl, H., Terpstra, P. \& Keck, W. (1991) FEMS Microbiol. Lett. $78,213-220$

6. Erpicum, T., Granier, B., Delcour, M., Lenzini, V. M., NguyenDistèche, M., Dusart J. \& Frère, J. M. (1990) Biotechnol. Bioeng. 35, 719-726

7. Granier, B. (1990) Ph.D. Thesis, University of Liège

8. Hopwood, D. A., Bibb, M. J., Chater, K. F., Kieser, T., Bruton, C. J., Kieser, H. M., Lydiate, D. J., Smith, C. P., Ward, J. M. \& Schrempf, H. (1985) Genetic Manipulation of Streptomyces: A Laboratory Manual, The John Innes Foundation, Norwich

9. Maniatis, T., Fritsch, E. F. \& Sambrook, J. (1982) Molecular
Cloning. A Laboratory Manual, Cold Spring Harbor Laboratory, Cold Spring Harbor, New York

10. Frère, J. M., Ghuysen, J. M., Reynolds, P. E., Moreno, R., Perkins, H. R., Dierickx, L. \& Delcambe, L. (1974) Biochem. J. 143, 241-249

11. Saiki, R. K., Gelfand, D. H., Stoffel, S., Scharf, S. J., Higuchi, R., Horn, G. T., Mullis, K. B. \& Ehrlich, H. A. (1988) Science 239, 487-491

12. Wallace, R. B., Johnson, N. J., Hirose, T., Miyake, M., Kawashima, E. H. \& Itakura, K. (1981) Nucleic Acids Res. 9, 879-894

13. Woods, D. (1984) Focus 6, 1-3

14. Sanger, F., Nicklen, S. \& Coulson, A. R. (1977) Proc. Natl. Acad. Sci. U.S.A. 74, 5463-5467

15. Staden, R. (1984) Nucleic Acids Res. 12, 551-567

16. Fickett, J. W. (1982) Nucleic Acids Res. 10, 5303-5318

17. Houba, S., Willem, S., Duez, C., Molitor, C., Dusart, J., Frère, J. M. \& Ghuysen, J. M. (1989) FEMS Microbiol. Lett. 65, 241-246

18. Frère, J. M., Leyh-Bouille, M., Ghuysen, J. M., Nieto, M. \& Perkins, H. R. (1976) Methods Enzymol. 45B, 610-636

19. Hanahan, D. (1983) J. Mol. Biol. 166, 557-580

20. Goad, W. B. \& Kanehisa, M. I. (1982) Nucleic Acids Res. 10, 247-263

21. Devereux, J., Haeberli, P. \& Smithies, O. (1984) Nucleic Acids Res. $12,387-395$

22. von Heijne, G. (1984) J. Mol. Biol. 173, 243-251

23. Eckhardt, T., Strickler, J., Gorniak, L., Burnett, W. V. \& Fare, L. R. (1987) J. Bacteriol. 169, 4249-4256

24. Dideberg, O., Charlier, P., Wéry, J. P., Dehottay, P., Dusart, J., Erpicum, T., Frère, J. M. \& Ghuysen, J. M. (1987) Biochem. J. 245, 911-919

25. Lamotte-Brasseur, J., Dive, G., Dideberg, O., Charlier, P., Frère, J. M. \& Ghuysen, J. M. (1991) Biochem. J. 279, 213-221

26. Korat, B., Mottl, H. \& Keck, W. (1991) Mol. Microbiol. 5, 675-684

27. Pratt, J. M., Jackson, M. E. \& Holland, I. B. (1986) EMBO J. 5, 2399-2405

28. Ghuysen, J. M., Reynolds, P. E., Perkins, H. R., Frère, J. M. \& Moreno, R. (1974) Biochemistry 13, 2539-2547

29. Frère, J. M. \& Joris, B. (1985) CRC Crit. Rev. Microbiol. 11, 299-396

30. Duez, C., Joris, B., Frère, J. M., Ghuysen, J. M. \& Van Beeumen, J. (1981) Biochem. J. 193, 83-86 